\title{
Review of: "Phosphoregulation of DSB-1 mediates control of meiotic double-strand break activity"
}

\author{
Akira Shinohara ${ }^{1}$ \\ 1 Osaka University
}

Potential competing interests: The author(s) declared that no potential competing interests exist.

The paper by Guo et al. describes the role of the phosphorylation of DSB-1 protein necessary for meioticDNA double-strand break (DSB) formation in C. elegans, which is regulated by a concerted action between DNA damage response kinases, ATM and ATR (atm-1 and atr-1 in C. elegans, respectively), and protein phosphatase, PP4 (pph-4.1 in C. elegans). The authors showed that DSB-1 protein is phosphorylated mainly by ATR-1 and de-phosphorylated by PPH-4.1. The phosphorylation down-regulates the ability of DSB-1 to introduce meiotic DSBs. Phosphorylation-defective dsb-1 mutant, such as dsb-1(5A), shows increased DSB levels in a wild-type background and rescues DSB defect in the pph-4.1 mutant. Moreover, the authors found that the dsb-1 (5A) allele can rescue reduced DSB formation in the mutant of the DSB-2, which is a paralog of DSB-1 in the nematode.

In the paper, the authors used cytological methods with western blotting analysis of the protein to analyze the role of DSB-1 phosphorylation and its regulator in C. elegans. The experiments were carried out wellcontrolled and the results are very much convincing. The paper would be written well and is easy to follow. Such structure analysis of DSB-1/Rec114 family protein with DSB-2 and DSB-3/Mei4 would be very informative.

On the other hand, as the authors acknowledged well, their results in inhibitory phosphorylation of DSB-1 for meiotic DSB formation are similar to those of the budding yeast ortholog of DSB-1, Rec114 (Carballo et al. PLoS Genetics, 2013). ATR(Mec1)-dependent multiple phosphorylation of Rec114 down-regulates DSB formation. In this line, the authors' results confirm the evolutionary conservation of regulatory networks for DSB formation. A novel observation in the paper is that the authors identified a counteracting protein phosphatase in the regulation, that is conserved PP4 phosphatase, which is predicted by a previous study of the authors' group (Sato-Carlton et al. PLoS Genetics, 2016) and the well-established relationship between Mec1/ATR and PP4 in various organisms.

To get some insight into the molecular mechanism of how the phosphorylation of DSB-1 suppressed DSB formation (through the localization or protein complex formation or others) as well as to know the role of pph-4.1 in DSB formation, the authors may need to address the following four major points. These points 
could be evaluted prior to publication based on discussion with editors in a journal.

1. The localization of DSB-1 protein on meiotic chromosomes has been described by two labs including coauthor's in this paper (Rosu et al. PLoS Genetics, 2016; Stamper et al. PLoS Genetics, 2016; Hinman et al. PNAS, 2021). It would be important to show the DSB-1 localization in pph-4.1 mutant as well as atr-1 depletion (or heterozygous) and the localization of DSB-1(5A) mutant protein in a different stage of meiosis.

2. As shown in yeast Rec114 (Carballo et al. PLoS Genetics, 2013), a phospho-memetic mutant of DSB-1 such as dsb-1 (5D or 5E) would be studied. Particularly, such a phospho-memetic mutant can phenocopy the pph-4.1 defect in DSB formation.

3. Given that the delayed RAD51-loading in the pph-4.1 mutant is not rescued by the atl-1 heterozygous (Fig. 1H) and dsb-1 (5A) (Fig. 2C), there could be other targets (s) of PPH-4.1 for "efficient and/or timely DSB formation". This point should be discussed. Importantly, the authors' argument described on page 3 , right column, 1st paragraph (last sentence) and page 4, left column, $3^{\text {rd }}$ paragraph is not convincing; a defect in post-DSB is unlikely to explain delayed DSB formation in the pph-4.1 mutant. If the DSB-1 phosphorylation negatively regulates DSB formation by feedback, one could imagine the pph-4.1 would affect only "late" formation, but not "early" DSB formation which is essential for the phosphorylation. These points should be more carefully discussed or could be studied by more experimentation.

4. From the end of page 4 to the beginning of page 5; Fig. 5C: The effect of worm aging on meiotic DSB formation sounds very interesting and worthwhile more analysis. It is good to show or refer that old worm gonad has more "spontaneous DSBs" or not (by looking at Rad51-staining in old spo-11 mutant etc), given more DSB-1 phosphorylation in old worms.

Minor points (not essential):

1. Page 2, right column, second paragraph: It would be important for readers to explain what the htp-3 and chk-2 genes are. Particularly, the role of chk-2 in C. elegans meiosis is quite different from a conserved role in the DNA damage response pathway.

2. Western blotting in Fig. 1A, Fig. 5C, Supplemental Fig. 1B, C, D: although the quantification is not essential, it would be important to mention the reproducibility of these results.

3. Figure 1B: In the typical dephosphorylation experiment, it would be better to add a phosphatase inhibitor in the treatment with the phosphatase as a control.

4. Figure 1D: It shows the gamma-ray-induced band shift of DSB-1 depending on ATR/ATM. But, because of two separated blots, it is hard to see the effect of atr-1 atm-1 mutation on band shift of Spo-11 induced band shift of the protein.

5. Figure 3B, C, and D: It would be nice to add the data in the dsb-1(5A) as a control.

6. Supplemental Fig. 1B: What did the authors mean by "pph-4.1(tm1598) balance heterozygous and homozygous mutants further treated with pph-4.1 RNAi"? Why the homozygous mutant was treated 
with RNAi? 